\title{
The Optimizations of MOSFET Contents in EE Undergraduate Course by using the Third Generation Semiconductor (Gallium Nitride)
}

\author{
Chen Yuanlong ${ }^{1}$ \\ ${ }^{1}$ Leeds Joint School, Southwest Jiao Tong University, Chengdu, Sichuan, 611756, China
}

\begin{abstract}
Recently, the third generation semiconductor----Gallium Nitride based electrical devices earn a more and more popular status in the industry for its easy popularization and cost effectivity. And another reason is the MOSFET with Gallium Nitride applied in power switching. However, transistors-related EE major (Electronic and Electrical engineering) courses are still focusing on the old silicon-based transistors, which own many deficiencies. In this paper, the current status of Gallium Nitride based MOSFET is investigated. Besides, a comparison in conducting capability, sensitivity and power efficiency between the MOSFET IRF510 and the Gallium Nitride based product GS-065-008-1-L is carried out. After the comparison, the application of MOSFET in EE courses is suggested and the priorities and difficulties are discussed as well.
\end{abstract}

\section{Introduction}

With the increasing demands of modern industrial appliances and products increase, transistors, especially MOSFET (Metal-Oxide-Semiconductor Field-EffectTransistor) are frequently used in the switching devices for its high conductivity, sensitivity and power efficiency. In order to broaden its application, GaN-based MOSFET should be introduced to more engineers, especially to the "future engineers" in university. Many EE (Electronic and Electrical Engineering) students in China are still studying outdated technologies and devices in the universities. In order to be well-prepared for the future work, they ought to access to the prevailing technology and appliances. Hence, the old contents in EE courses should be replaced by the new contents of GaN MOSFET.

In this paper, the optimization of MOSFET contents in EE undergraduate courses has been suggested. Firstly, a review of the current status of GaN MOSFET is presented. Secondly, a review of current used n-channel MOSFET 'IRF510' and a brief introduction of a typical Gallium Nitride based n-channel MOSFET 'GS-065-008-1-L' will be made to compare, which are within the aspects of conducting capability, sensitivity and power efficiency to show the deficiencies of old version transistor (IRF510). In this case, the suggested replacement of the MOSFET used in EE courses will be taken as the optimization of EE undergraduate courses. In addition, a further discussion about the difficulties to achieve the replacement by now is included.

\section{Current status of gallium nitride MOSFETS}

Corresponding E-mail: angela@cas-harbour.org
In the past, Gallium Nitride (GaN) technology was introduced as an excellent alternative to MOSFET. As the industry developed, Gallium Nitride was assumed as an innovative technology to replace the aging power MOSFET. Nevertheless, by the selection of any leading technologies two factors must be taken into consideration: the popularization and the cost.

Easy popularization. Gallium Nitride transistors behave similarly to aging power MOSFET devices in principles and practice. In order to popularize this new material MOSFET in the area like power convention, some companies come out some solutions that engineers and students in this major can get familiar with the use of Gallium Nitride devices and related applications easily. Take the company 'EPC' as an example [5].

Firstly, in terms to engineers, EPC has published the industry's first textbook on Gallium Nitride transistors for help, both in English and in simplified Chinese, Efficient Conversion Devices -- Gallium Nitride Transistors. Recently, they published two handbooks on hot applications to help power design engineers use Gallium Nitride devices in the application of DC/DC conversion and wireless power transmission systems.

Secondly, as to students, EPC is currently working with more than 60 universities around the world to provide the next generation of experienced power system design engineers with training in gallium nitride technology to further enhance their design experience to maximize the benefits of gallium nitride technology.

Cost-effective. In industry productions, Gallium Nitride devices are manufactured with similar process to silicon-based MOSFET but in fewer steps and can yield more devices per manufacturing process, which is due to the much smaller size of Gallium Nitride products. In addition, the lower voltage Gallium Nitride transistors 
(below $500 \mathrm{~V}$ ) do not require the more expensive packaging of equivalent silicon-based devices. This packaging advantage alone has halved the manufacturing cost of gallium nitride devices, plus its advantages of high

\begin{tabular}{l|c|c|} 
& $\mathbf{2 0 1 4}$ & $\mathbf{2 0 1 6}$ \\
\hline Starting Material & lower & lower \\
\hline Epi Growth & same & lower \\
\hline Wafer Fab & same & lower \\
\hline Test & same & same \\
\hline Assembly & lower & lower \\
\hline OVERALL & lower & lowerl \\
\hline
\end{tabular}

manufacturing yield and small size, allowing Gallium Nitride Wafer fab cheaper but their devices more powerful [5].

\begin{tabular}{|l|c|c|}
\hline & $\mathbf{2 0 1 4}$ & $\mathbf{2 0 1 6}$ \\
\hline Starting Material & lower & lower \\
\hline Epi Growth & higher & rame \\
\hline Wafer Fab & same & lower \\
\hline Test & same & same \\
\hline Assembly & lower & lower \\
\hline OVERAL & higher & lowerl \\
\hline
\end{tabular}

Fig. 1. Equivalently-rated GaN Transistor products' cost compared to silicon MOSFET [1].

From Fig.1, in 2014, GaN transistors owned the same or even higher cost than silicon MOSFET due to the relatively low level of equipment utilization. But until 2016, invented enhancement-mode GaN transistors needed fewer steps to manufacture, which low down the overall cost.

Consequently, the current status of Gallium Nitride MOSFETS shows their "winning advantages" in industry applications, which including easy popularization and extremely cost-effective to silicon-based MOSFET. Gallium Nitride technology is relatively new and it has huge potential for development. That's why gallium nitride was chosen and why it owns such a popular status.

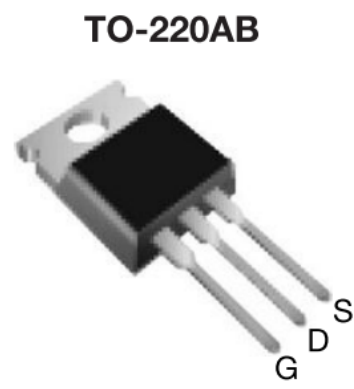

\section{IRF510 \& GS-065-008-1-L Comparison and Analysis}

Introduce of EE course using MOSFET----IRF510. A course called Circuit Analysis and Design is generally taken by the most of the EE students around world. This module provides an introduction to the key electronic components by taking classes and labs, which are the basic concepts of electronic circuit design and the basic principles of electronic circuit test and measurement. For further details about key electronic components, transistors are frequently used in most of the classes and labs, which are especially MOSFETs for controlling the passing current and voltage in the circuit.

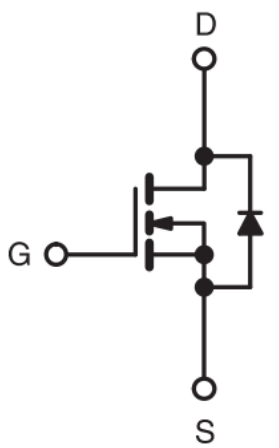

N-Channel MOSFET

Fig. 2. N-Channel MOSFET-IRF510 inside circuit (right) and component's outlook (left) [3].

Take SWJTU-Leeds Joint School as an example, in the sophomore year, EE undergraduate course 1130 (Circuit analysis and design) practically provides n-channel MOSFETs 'IRF510' to do most of the labs related to transistors. In order to understand the characteristics and circuit operation of field-effect transistors and be able to apply them in simple circuits, the datasheet of the IRF510 MOSFETs is given, which makes much help to do the following comparison.

Here is using IRF510, third generation Power
MOSFETs from Vishay, which used to be considered as the item provides the designer best combination of fast switching, ruggedized device design, low on-resistance and cost-effectiveness, is based on silicon made transistor, which silicon materials belong to the first-generation semiconductors. So IRF510 is a typical type of firstgeneration semiconductor made transistor [3]. 

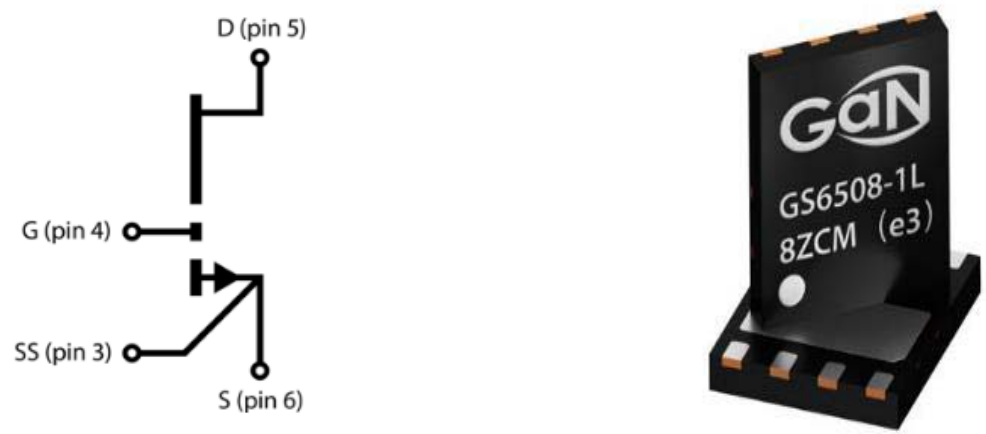

Fig. 3. GaN based MOSFET--GS-065-008-1-L inside circuit (left) and component's outlook (right) [2].

Introduce of Gallium Nitride based MOSFET---GS-065-008-1-L. Third generation semiconductor, as mentioned in previous introduction, is famous for its efficiently applications. As one of the typical materials of the third-generation material semiconductor, Gallium Nitride, which is the compound of the Gallium and Nitrogen, is well-known for its wide bandgap of $3.4 \mathrm{eV}$. With such wide bandgap, more energy is needed to excite such semiconductor to conduct, which means this new type of semiconductor can hold higher voltage and current. With these advantages, Gallium Nitride is widely used in power switching devices frequently. Compared with the first-generation material (silicon-based) semiconductor, Gallium Nitride makes more significant improvement in conducting capability, sensitivity and thermal resistance, which provide very high efficiency power switching. Further data of these characteristics are given by the data sheet offered by the products' page on the website of GaN system, which makes help to the following comparison of two types of transistors.

Here is using GS-065-008-1-L, the enhancement mode GaN-on-silicon power transistor, is an n-channel

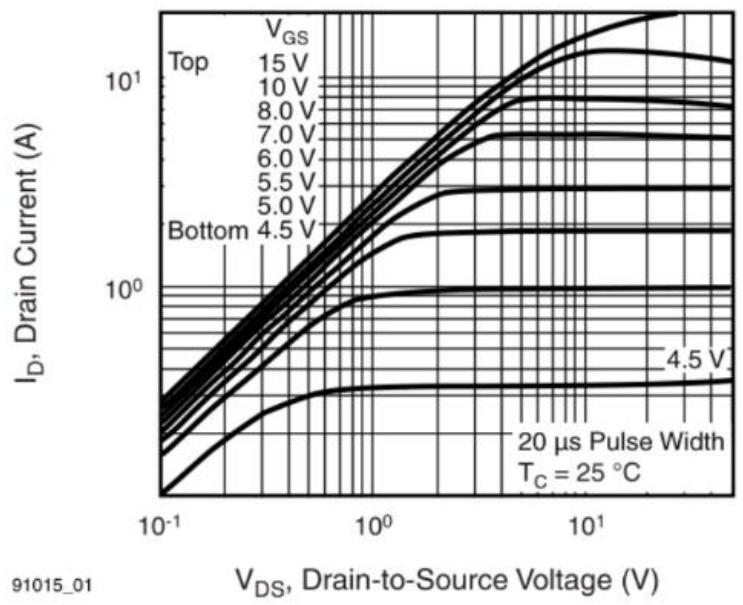

MOSFET. It allows $650 \mathrm{~V}$ drain-source breakdown voltage and $8 \mathrm{~A}$ continuous drain current [2].

Comparison and analysis (From switches' perspective). Evaluating the performance of n-channel Si based IRF510 MOSFET and GaN based MOSFET requires study and analysis of graphs of some experimental characteristics. From the perspective of MOSFET that it is frequently used for switches in electrical devices, the conducting capability, sensitivity and power efficiency are mainly focusing on. Due to the comparison towards these three main functions, the deficiencies of using IRF510 in recent EE courses will be analyzed and illustrated.

For conducting capability, switching devices are mainly focus on the transistors' properties that about voltage \& current carrying ability, especially for its maximum drain current and maximum drain-source voltage at a certain temperature. Here 25 degree has been settled to be the certain temperature condition, and the further data of currents and voltages at 25 degrees are all picked out from datasheets of two type of transistors and shown as follow.

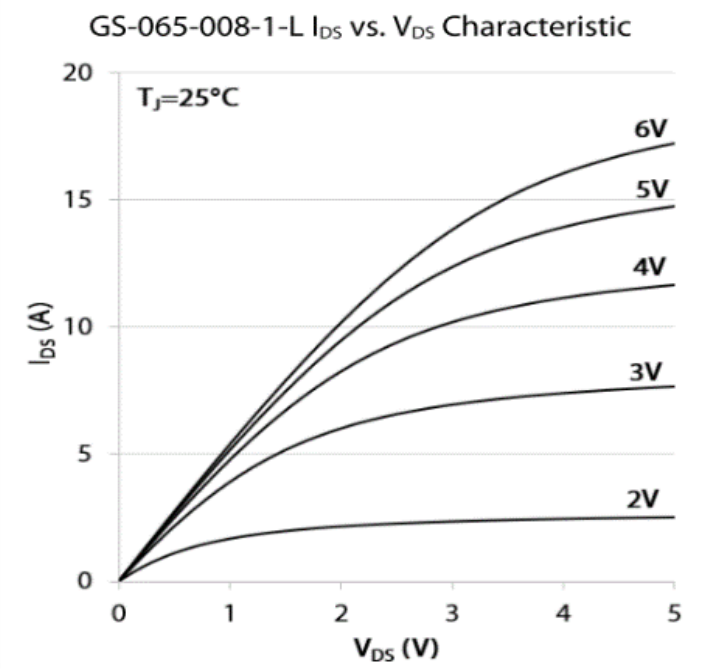

Fig.4. Ids/Vds of IRF510 [3] (left) and Ids/Vds of GS-065-008-1-L [2] (right).

From Fig.4, as comparison of two Ids/Vds graphs under 25 degrees with certain values of Vgs and Vds, the maximum carrying currents 'Ids' are obvious to be seen the difference. Take $\mathrm{Vds}=5 \mathrm{v}$ and $\mathrm{Vgs}=6 \mathrm{v}$ as an example. Within this condition, IRF510 shows a maximum carrying current about 5A [3], while GS-065-008-1-L shows an approximate maximum carrying current more than $15 \mathrm{~A}[2]$, which is at least three times more than IRF510. As the result of that, within the same conditions, GS-065-008-1L shows a better performance in high current carrying than 
IRF 510

\begin{tabular}{|c|c|c|c|}
\hline PARAMETER & SYMBOL & LIMIT & UNIT \\
\hline Drain-Source Voltage & $V_{D S}$ & 100 & v \\
\hline
\end{tabular}

Fig. 5. The limit carrying Drain-Source Voltage of IRF510 at $25^{\circ} \mathrm{C}[3]$.

\begin{tabular}{|c|c|c|c|}
\hline Drain-to-Source Voltage & $V_{D S}$ & 650 & $\mathrm{~V}$ \\
\hline Drain-to-Source Voltage - transient (note 1) & $V_{D S(t r a n s i e n t)}$ & 750 & V \\
\hline
\end{tabular}

Fig. 6. The limit carrying Drain-Source Voltage of GS-065-008-1-L at $25^{\circ} \mathrm{C}$ [2].

From Fig.5 and Fig.6, which is about the maximum carrying voltage at 25 degrees, IRF510 shows the maximum drain-source voltage of $100 \mathrm{v}$ [3], while GS065-008-1-L shows the maximum drain-source voltage of $650 \mathrm{v}$ and transient $750 \mathrm{v}$ [2], which is approximately 6.5 7.5 times higher than IRF510. As the result of that, at the same temperature, GS-065-008-1-L shows a better performance in high voltage carrying than IRF510.

For sensitivity, switches' reacting time is typically

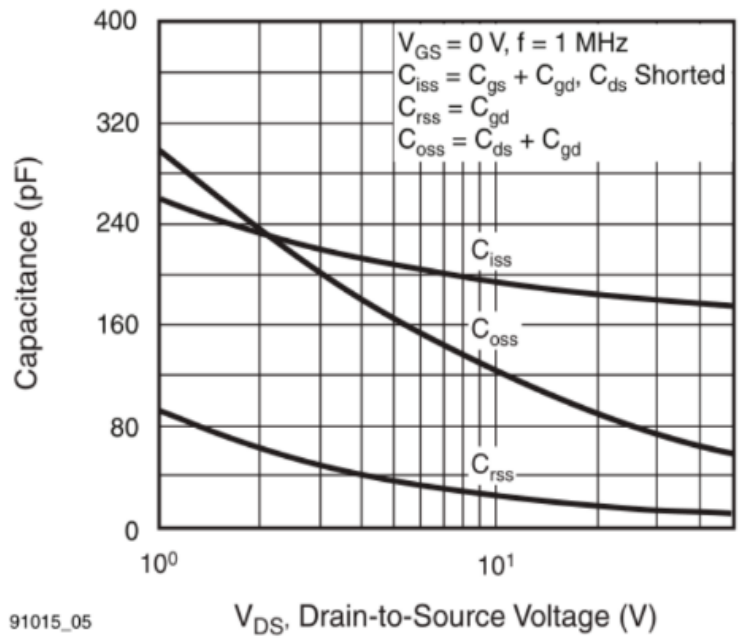

described by their property of its capacitance within a certain range of drain-to-source voltage. Due to the charging equation:

$\mathrm{Vt}=\mathrm{E} *[1-\exp (-\mathrm{t} /(\mathrm{R} * \mathrm{C}))] \quad \rightarrow \quad \mathrm{t}=\mathrm{R} * \mathrm{C} * \ln [\mathrm{E} /(\mathrm{E}-\mathrm{Vt})] \quad$ (1)

It is shown that higher capacitance causes longer reacting time, lower capacitance transistors earn more demands by products.

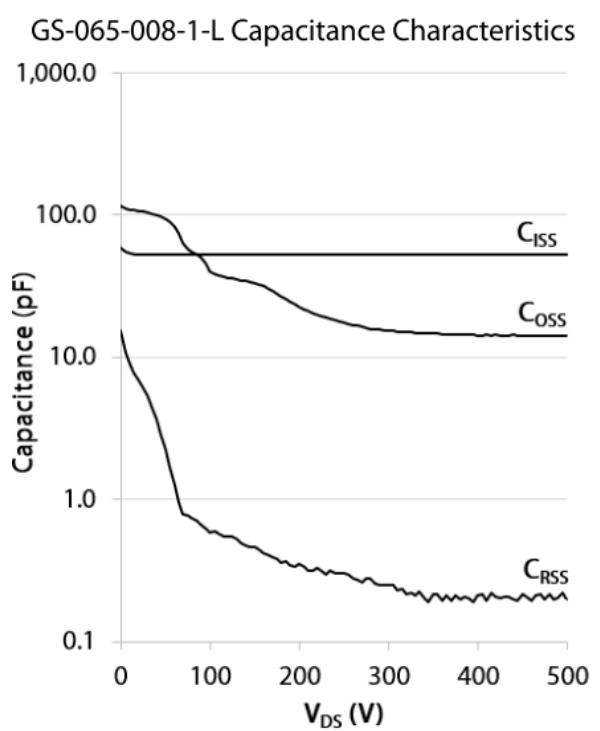

Fig. 7. Typical capacitance of IRF510 [3] (left) and capacitance of GS-065-008-1-L (right) [2].

As observation from Fig. 7 above, take the Vds range $0-10 \mathrm{v}$ as the observing condition. Ciss and Coss of IRF510 are approximately 120pf to 300pf [3], while for GS-065008-1-L are around 100pf [2], which are 1.2-3 times smaller than IRF510. Meanwhile, Crss of IRF510 is about $30 \mathrm{pf}$ to $90 \mathrm{pf}$ [3], while for GS-065-008-1-L is about 10pf [2], which is 3 to 9 times smaller than IRF510. As the result of that, within a certain Vds range, GS-065-008-1-L shows smaller capacitance than those of IRF510, which also means GS-065-008-1-L needs fewer reacting time than IRF510 at the same Vds condition. In this case, GS065-008-1-L owns a higher sensitivity than IRF510.

For switches, another important function that need considering is the power efficiency of them. Among all of the effective factors of the power efficiency, the heat dissipation is the most influencing factor of the power efficiency for an electrical device. In this case, transistors' property 'Thermal resistance' of two types of transistors is mentioned and compared here. Thermal resistance refers to the ratio of the temperature difference between the two ends of a body and the power of a heat source when heat is transferred over it. The units are kelvin per watt $(\mathrm{K} / \mathrm{W})$ or Celsius per watt $\left({ }^{\circ} \mathrm{C} / \mathrm{W}\right)$. High thermal resistance means higher heat dissipation caused by each watt of power from the conducting current. In this case, power switching transistors with lower thermal resistance earn more demands for products.

\section{Thermal Resistance}

\begin{tabular}{|c|c|c|c|c|c|}
\hline & Parameter & Min. & Typ. & Max. & Units \\
\hline$R_{\text {QuC }}$ & Junction-to-Case & - & - & 3.5 & \multirow{3}{*}{${ }^{\circ} \mathrm{C} / \mathrm{W}$} \\
\hline$R_{\theta C S}$ & Case-to-Sink, Flat, Greased Surface & - & 0.50 & - & \\
\hline RANA & Junction-to-Ambient & - & - & 62 & \\
\hline
\end{tabular}

Fig. 8. Typical thermal resistance of IRF510 [3]. 
Thermal Characteristics (Typical values unless otherwise noted)

\begin{tabular}{|l|c|c|c|}
\hline Parameter & Symbol & Value & Units \\
\hline Thermal Resistance (junction-to-case) - bottom side & $R_{\ominus \mathrm{AC}}$ & 2.0 & ${ }^{\circ} \mathrm{C} / \mathrm{W}$ \\
\hline Thermal Resistance (junction-to-ambient) (note 2) & ReJA & 38 & ${ }^{\circ} \mathrm{C} / \mathrm{W}$ \\
\hline
\end{tabular}

Fig. 9. Typical thermal resistance of GS-065-008-1-L [2].

As comparison between Fig. 8 and Fig. 9 above, IRF510 shows the maximum junction-to-case thermal resistance of $3.5^{\circ} \mathrm{C} / \mathrm{W}[3]$, while GS-065-008-1-L shows a lower junction-to-case thermal resistance of $2.0^{\circ} \mathrm{C} / \mathrm{W}$ [2]. Meanwhile, IRF510 shows the maximum junction-toambient thermal resistance of $62^{\circ} \mathrm{C} / \mathrm{W}[3]$, whereas GS065-008-1-L shows a lower junction-to-ambient thermal resistance of $38^{\circ} \mathrm{C} / \mathrm{W}$ [2]. As the result of that, GS-065008-1-L shows almost a half lower thermal resistance than IRF510, which means GS-065-008-1-L shows a higher power efficiency than IRF510 from the perspective of the factor about heat dissipation.

\section{Discussion}

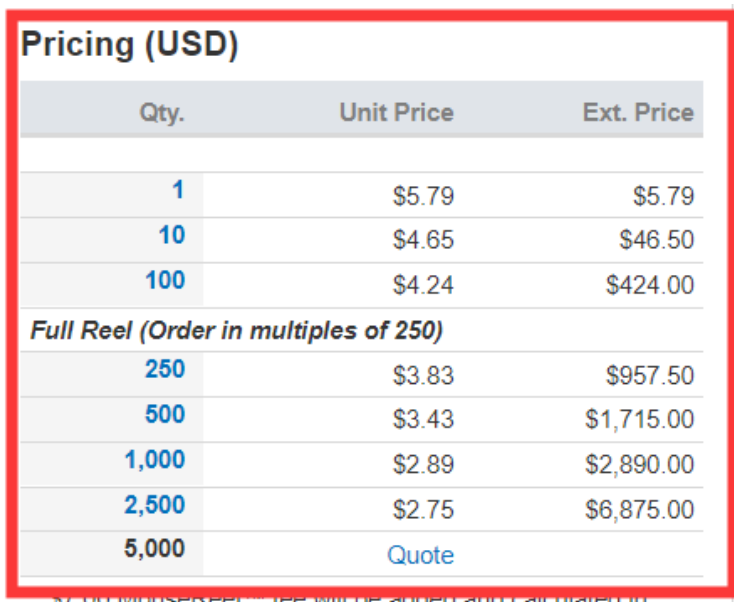

Fig. 10. Pricing of IRF510[7].
For the popularization of the $\mathrm{GaN}$ based MOSFET in school, there are actually some difficulties for students to get known about this new material. First of all, it may generate parasitic inductance in the circuit [4], which students have less experience to minimize these unwanted parasitic and enable a much more efficient layout, less voltage overshoot, and generates much less unwanted noise. Secondly, the smaller size of GaN devices requires tighter assembly tolerances more similar to those used in computer and telecom equipment but less familiar to undergraduate students. In this case, more related knowledge of using GaN based MOSFET need to be offered by the major courses.

\begin{tabular}{|rrr|}
\hline Pricing (USD) & & \\
\hline Qty. & Unit Price & Ext. Price \\
\hline 1 & $\$ 0.88$ & $\$ 0.88$ \\
\hline 10 & $\$ 0.73$ & $\$ 7.30$ \\
\hline 100 & $\$ 0.605$ & $\$ 60.50$ \\
\hline 500 & $\$ 0.506$ & $\$ 253.00$ \\
\hline 2,500 & $\$ 0.343$ & $\$ 857.50$ \\
\hline 5,000 & $\$ 0.309$ & $\$ 1,545.00$ \\
\hline 10,000 & $\$ 0.297$ & $\$ 2,970.00$ \\
\hline 25,000 & $\$ 0.286$ & $\$ 7,150.00$ \\
\hline
\end{tabular}

Fig. 11. The price of GS-065-008-1-L from Mouser Electronics[6].

analysis above.

For the costs from Fig.10 and 11, GS-065-008-1-L owns almost seven times higher price than IRF510. The reason is although cost-effective function of GS-065-0081-L has been proved in the earlier paragraphs, it is only in terms of industrial-strength MOSFET, while IRF510 is less frequently used in modern industrial productions because of its low efficiency but low cost. In this case, it causes that GS-065-008-1-L owns lower competitiveness than IRF510 from the perspective of universities' EE educational usage. However, in order to teach advanced GaN MOSFET to EE students, higher cost of optimizing the MOSFET with GaN based version is worthy for EE students to be prepared to advanced knowledge and future work.

Derived from analysis above, actually it is not only for the MOSFET items, but also can be anything else about the electrical devices. Therefore, it should be raised up to the view of all of the electrical items used in courses, which can all be optimized by GaN based devices from the

\section{Conclusion}

In this paper, an introduction of status that choosing Gallium Nitride for MOSFET and a comparison between IRF510 and GS-065-008-1-L power switches are presented. The current status of Gallium Nitride mainly shows the advantage of $\mathrm{GaN}$ in easy popularization and cost-effective. Moreover, comparisons between the EE course used MOSFET IRF510 and an industrial used GaN MOSFET GS-065-008-1-L are introduced. The comparisons show the deficiencies of using IRF510 from perspectives of capability, sensitivity and power efficiency.

Based on all the above, it is suggested to replace silicon based IRF510 MOSFET with Gallium Nitride based MOSFET in the courses for EE students, so that the student can obtain more practical and advanced knowledge and make better preparation for the future 
work. However, the difficulty in popularization of the new product in universities and the cost for universities limit the realization of this conception. To solve this problem, other devices that haven't been based on third-generation semiconductor should be developed and optimized.

\section{References}

1. A. Lidow: GaN transistors - Giving new life to Moore's Law, Proc. Int. Symp. Power Semicond. Devices ICs. Vol. 2015-June (2015), p. 1-6.

2. GaN System (Company) : "GS66508P Bottom-side cooled $650 \mathrm{~V}$ E-mode GaN transistor Preliminary Datasheet GS66508P Bottom-side cooled 650 V Emode GaN transistor Preliminary Datasheet Absolute Maximum Ratings ( $\mathrm{T}$ case $=25^{\circ} \mathrm{C}$ except as noted ) Parameter Parameter Ordering Informati," (2018), p. $1-16$.

3. V. Siliconix: IRF510, SiHF510, no. V

4. K. J. Chen et al.: GaN-on-Si power technology: Devices and applications, IEEE Trans. Electron Devices. Vol. 64, no. 3 (2017), p. 779-795.

5. Retrieved from (2019.12.11): https://epcco.com/epc/GalliumNitride/whygan.aspx

6. Retrieved from (2019.12.11): https://www.mouser.com/ProductDetail/GaNSystems/GS-065-008-1L?qs $=$ sGAEpiMZZMshyDBzk1\%2FWi\%2FD7Em 5shE8q9fUamhl6LWFLq1Q8\%252BucJWw==

7. Retrieved from (2019.12.11): https:/www.mouser.com/ProductDetail/VishaySiliconix/IRF510PBF?qs=sGAEpiMZZMshyDBzk 1\%2FWi1F3z9PgzPBnjAas2zQPJco\%3D 